\title{
CURADURÍA E IDENTIFICACIÓN DE LA FAUNA DE Bombus DE LA COLECCIÓN DE ENTOMOLOGÍA DE LA UNIVERSIDAD DE CIENCIAS APLICADAS Y AMBIENTALES U.D.C.A
}

\section{CURATION AND IDENTIFICATION OF Bombus FAUNA DEPOSITED AT U.D.C.A'S ENTOMOLOGICAL COLLECTION}

\author{
Lorena Téllez-Farfán ${ }^{1}$, Francisco J. Posada-Flórez²
}

\begin{abstract}
${ }^{1}$ Estudiante Fac. Ciencias Ambientales. Joven investigadora. Universidad de Ciencias Aplicadas y Ambientales U.D.C.A, Calle 222 No.55-30, Bogotá, D.C. Colombia, e-mail: tllezlorena@gmail.com; ${ }^{2}$ Ing. Agrónomo, Entomólogo Ph.D. Profesor investigador Universidad de Ciencias Aplicadas y Ambientales U.D.C.A, hasta Junio 2012. e-mail: fjavierposada@hotmail.com
\end{abstract}

Rev. U.D.C.A Actr. \& Div. Cient. 16(2): 501-509, Julio-Diciembre, 2013

\section{RESUMEN}

Las colecciones de insectos son una herramienta de educación, extensión e investigación, que requieren el mantenimiento y la curaduría, para que la información contribuya al conocimiento y a la conservación de la biodiversidad de las especies colectadas. En este trabajo, se tuvo como objetivo realizar la curaduría e identificación de las especies de Bombus, depositadas en la colección entomológica de la U.D.C.A. Se encontraron ocho especies, distribuidas en seis departamentos, con procedencia de 55 localidades ubicadas en ambas vertientes de las cordilleras oriental y central, a altitudes desde los 178 hasta los 3000msnm. Se reconocieron 25 plantas hospedantes para las especies $B$. atratus, $B$. robustus y $B$. rubicundus. Dentro de las plantas visitadas, se registró la mayor frecuencia de visitas por $B$. atratus sobre la mora silvestre y el trébol. El estado de curaduría de la colección de Bombus alcanzó el nivel de nueve y con el material organizado, se prepararon publicaciones, con el propósito de contribuir al mayor aprovechamiento y conservación de este grupo de polinizadores nativos.

Palabras clave: Abejorros, polinizadores, plantas hospedantes, distribución.

\section{SUMMARY}

Insect collections are tools for education, extension and research and require maintenance and curation in order to generate useful information contributing to the knowledge and conservation of the biodiversity of collected species. The objective of this study was to curate and identify the Bombus species deposited at U.D.C.A's entomological collection. Eight species of Bombus were identified from six Colombian departments distributed in 55 localities around the slopes of the eastern and central range of the Andean mountains, at altitudes oscillating from 178 to 3000 meters above sea level. Twenty-five host plants were found visited by $B$. atratus, $B$. robustus y $B$. rubicundus. Among visited plants, the highest frequency of visits by $B$. atratus was recorded on blackberry and clover flowers. The Bombus collection was appraised at a curation level of nine (out of ten) and the material was used to publish scientific papers contributing to a better understanding and conservation of this group of native pollinators.

Key words: Bumblebee, pollinators, host plants, distribution.

\section{INTRODUCCIÓN}

Las colecciones de insectos son una herramienta básica para la docencia en las universidades, con facultades donde se enseñan cursos de entomología, sea de tipo agrícola, veterinaria, médica o forense. Actualmente, se puede hablar de la "entomología ambiental", por la urgencia que hay de desarrollar programas para medir impactos ambientales, dirigidos a conocer la biodiversidad, establecer programas de conservación y remediación de hábitats amenazados por la 
actividad humana, de alto impacto negativo sobre el medio, donde los insectos, por su diversidad, taxonomía y funciones, son utilizados para adelantar estudios e investigaciones (Chung, 1993; Klein et al. 2007; James, 2010; Park et al. 2010). Además, las colecciones juegan un papel muy importante, como referencia en los centros de educación e investigación agrícola, donde millones de hectáreas de cultivos requieren mantener un conocimiento de los insectos plagas, enemigos naturales y polinizadores (benéficos) y una vigilancia permanente para estar atentos a tomar medidas de manejo sobre las explosiones de las poblaciones o de cuando ocurre la introducción de una nueva plaga invasora, que amenaza los cultivos y a los animales, como los polinizadores y a la economía de los agricultores y el país (Hernández et al. 1995; Cruz \& Posada, 2003).

Dentro del grupo de insectos de mayor importancia para el mantenimiento de la vida en la tierra están los polinizadores, porque con su función contribuyen a asegurar la reproducción de una tercera parte de la comida que utilizan los humanos y la producción de semillas y frutos para la alimentación de otro grupo de animales, vertebrados e invertebrados, en la cadena alimenticia que, adicionalmente, contribuyen a la repoblación de la diversidad de plantas (Nates \& Gonzáles 2000; Allsopp et al. 2008).

Dentro del grupo de los polinizadores, se encuentran las abejas del género Bombus (Hymenoptera: Apidae: Bombini), conocidos popularmente como abejorros. Este grupo de insectos, en el mundo, tiene 239 especies, que pertenecen a 28 géneros (Michener, 2007). En Colombia, esta fauna está representada por nueve especies nativas (Liévano et al. 1994).

El conocimiento de esta fauna es importante, debido a su función polinizadora de flora, aprovechada por el ser humano, como es el caso de algunas especies de la familia Solanaceae y porque las poblaciones de la abeja melífera (Apis mellifera), otro representante importante de la fauna polinizadora por excelencia, están siendo sujetas al ataque de plagas y de enfermedades, tanto de origen biótico como abiótico y a la pérdida del hábitat que está diezmando sus poblaciones y que se conoce como la muerte súbita de la colonia (CCD = colony collapse disorder) (Brown \& Paxton, 2009). Al igual que la abeja melífera, las poblaciones de Bombus y otras abejas nativas están sujetas a amenazas similares, lo que puede afectar severamente la biodiversidad y los servicios de polinización (Otterstatter \& Thomson, 2008).

Los Bombus viven en colonias de unos cientos de individuos; son una fauna silvestre sobre la que todavía se requiere realizar mucha investigación en nuestro medio, para mantenerlos y utilizarlos en la polinización de cultivos. Estos insectos no almacenan miel para aprovechamiento humano, pero son polinizadores muy eficientes, debido a su capacidad de zumbar las flores de ciertas plantas, para el desprendimiento del polen (Delaplane \& Mayer 2000; Almanza, 2007; Aldana et al. 2007; Cruz et al. 2008).

La información sobre la presencia de Bombus en el país mayormente proviene de los inventarios que se han realizado en colecciones (Nates \& Gonzáles 2000; Liévano et al. 1994), de unos pocos trabajos de colección en campo (MoralesRozo et al. 2006; Nates-Parra et al. 2006) y de la evaluación como agentes de polinización en cultivos como lulo y tomate (Almanza, 2007; Aldana et al. 2007). En Colombia, las especies de Bombus están amenazadas por la pérdida del hábitat, debido al auge de la urbanización, a la pérdida de la vegetación en los humedales, a la destrucción de los páramos, por las prácticas inadecuadas de manejo de la flora y las actividades agrícolas de uso intensivo de insecticidas y herbicidas para la destrucción de las malezas, que hacen parte de la flora que brinda los recursos néctar y polen, los cuales, son la base de la alimentación de Bombus.

Por la importancia de la fauna de Bombus en la polinización, las amenazas a sus poblaciones, por parte de las actividades humanas y la escasa información sobre esta fauna en el país, en este trabajo se tuvo por objetivo realizar la curaduría de las especies de Bombus presente en la colección de insectos de la Universidad de Ciencias Aplicadas y Ambientales U.D.C.A, en Bogotá, Colombia, identificar las especies presentes, conocer las plantas hospedantes, identificar la distribución y, con base en esta información, lograr plantear nuevas propuestas de investigación, tendientes a mejorar el conocimiento de esta fauna en el país, hacer un mejor aprovechamiento en la polinización de los cultivos y trazar planes de conservación.

\section{MATERIALES Y MÉTODOS}

Se revisó la colección de insectos de la U.D.C.A, contenida en los gabinetes y cajas con material montado en alfileres y la colección contenida en viales con alcohol. El material depositado en la colección ha sido colectado por los estudiantes de la asignatura de Entomología, como requisito de la parte práctica del curso.

El material colectado de Bombus, encontrado en las diferentes cajas de los gabinetes y en alcohol, se separó de otras abejas nativas, con las que presenta similitud. En la identificación, se utilizaron las características diagnóstico presentes en este grupo, según Abrahamovich \& Díaz (2002), Liévano et al. (1994), Tripplehorn \& Johnson (2005) y Michener (2007). Posteriormente, el material se separó y se evaluó el estado de conservación, siguiendo la escala de curaduría propuesta por McGinley (1993) y Cruz \& Posada (2003), para 
el manejo de colecciones. En la evaluación, se revisó el estado de preservación de cada espécimen, para establecer si se encontraba completo, si le faltaban partes o si presentaba ataque de plagas u hongos. También, se estimó la información contenida en las etiquetas, que acompañan al espécimen, para establecer la localidad, la fecha de colección, la forma de colección (si estaba libando en una flor y el nombre de la planta hospedante o si se colectó al vuelo) y, finalmente, el nombre del colector.

La información de la evaluación de la conservación de especímenes y la información contenida en las etiquetas, se transfirió a una hoja de cálculo de Excel con todas las entradas, para cada espécimen, colocadas en columnas correspondientes a la evaluación descrita anteriormente y se calcularon las estadísticas descriptivas. Se prepararon las gráficas para representar los resultados del estado de conservación de la colección de Bombus de la U.D.C.A. Terminada la evaluación, se utilizó la información para preparar nuevas etiquetas con datos que contenía las etiquetas originales y complementando la faltante, con la identificación y el estado de curaduría de los especímenes.

Con la información de localidad, se estableció la procedencia de los especímenes y la distribución, tanto geográfica como altitudinal, para la cual, se tomó como referencia la cabecera municipal. Las altitudes registradas, se dividieron en rangos de $1000 \mathrm{msnm}$, con el propósito de establecer la abundancia por clima cálido, medio y frío. Se preparó un mapa de distribución de las especies, tomando como base los especímenes que contenían en su ficha de colecta el municipio del que provenían. Se utilizó el programa ArcGis 9.3 (ESRI).

Igualmente, se evaluó la información sobre la forma de captura, para establecer las plantas hospedantes. En este trabajo, se dan recomendaciones para realizar la colección de especímenes de Bombus, para que la información de colecta tenga valor para la investigación y la conservación de este grupo de polinizadores.

La clasificación, se realizó siguiendo las características diagnóstico propuestas por Abrahamovich \& Díaz (2002), Liévano et al. (1994), Michener (2007) y Tripplehorn \& Johnson (2005) y utilizando la genitalia y cámara del aguijón de las hembras (Prŷs-Jones \& Corbet, 1991; Liévano et al. 1994; Téllez \& Posada, en preparación). Las genitalias extraídas, se conservaron en viales y se tomaron fotografías, utilizando un estereoscopio, con el propósito de registrar y documentar fotográficamente las especies y las características diagnóstico, para utilizarlas con fines de investigación y educación en la conservación de Bombus.

\section{RESULTADOS Y DISCUSIÓN}

En la colección de insectos de la U.D.C.A, se encontraron 213 especímenes de Bombus, de los cuales, 179 (84,0\%) arrojaron información, que permitió establecer el departamento y la localidad donde se colectaron. De este material colectado, 34 (16,0\%) especímenes no presentaron ningún dato de valor para el conocimiento del género, lo que significa una pérdida de oportunidad del conocimiento de la biodiversidad de los lugares donde se colectaron estos especímenes y no permite establecer la relación con la flora que les sirve de sustento.

En la colección, los especímenes de Bombus provinieron de seis departamentos y 55 localidades o municipios colombianos. Por departamento, el mayor número de colectas de Bombus se realizó en Cundinamarca, con 149 especímenes (83,2\%), de 30 localidades (64,0\%), seguido por Boyacá, con nueve especímenes (5,0\%), de nueve localidades (16,0\%); Tolima, con 12 especímenes (6,7\%), de cinco localidades $(9,1 \%)$; Meta, con cinco especímenes $(2,8 \%)$, de cuatro localidades (7,3\%); Casanare, con dos especímenes $(1,1 \%)$, de una localidad $(1,8 \%)$ y, el Valle del Cauca, con dos especímenes (1,1\%) (Figura 1A). Esta fauna de Bombus corresponde a la región central de Colombia, con distribución en ambos flancos de la cordillera oriental y central.

En la identificación de los especímenes de Bombus de la colección de la U.D.C.A, se encontraron siete especies y una morfo-especie, utilizando el patrón de coloración y la disección de especímenes, por la extracción de la genitalia y la cámara del aguijón (Abrahamovich \& Díaz, 2002; Liévano et al. 1994; Michener, 2007; Téllez \& Posada, en preparación).

En la figura $1 \mathrm{C}$, se presenta, para cada especie de Bombus, la distribución por departamento y por municipio. Tomando como base aquellos especímenes que en su ficha de colecta presentaban esta información, se obtuvo la distribución de seis especies y la morfoespecie en cinco departamentos, ubicados en la zona central de Colombia: Casanare, Boyacá, Cundinamarca, Tolima y Meta. La mayor cantidad de información de distribución por especies, se encuentra en Cundinamarca, de donde Bogotá D.C. es el lugar con el mayor número de especies (Figura 1C).

$B$. atratus fue la especie que presentó mayor número de colectas (73,7\%); se colectó en los seis departamentos y en 45 localidades (81,8\%) (Figura 1B), lo que concuerda con los datos de distribución mencionados por Nates-Parra (2005), en donde se califica a $B$. atratus como la especie más abundante y con mayor distribución del género en Colombia, de lo que se podría inferir que es una especie flexible a los cambios en su entorno. $B$. robustus fue la segunda especie con mayor número de especímenes $(n=20,9,5 \%)$; se co- 

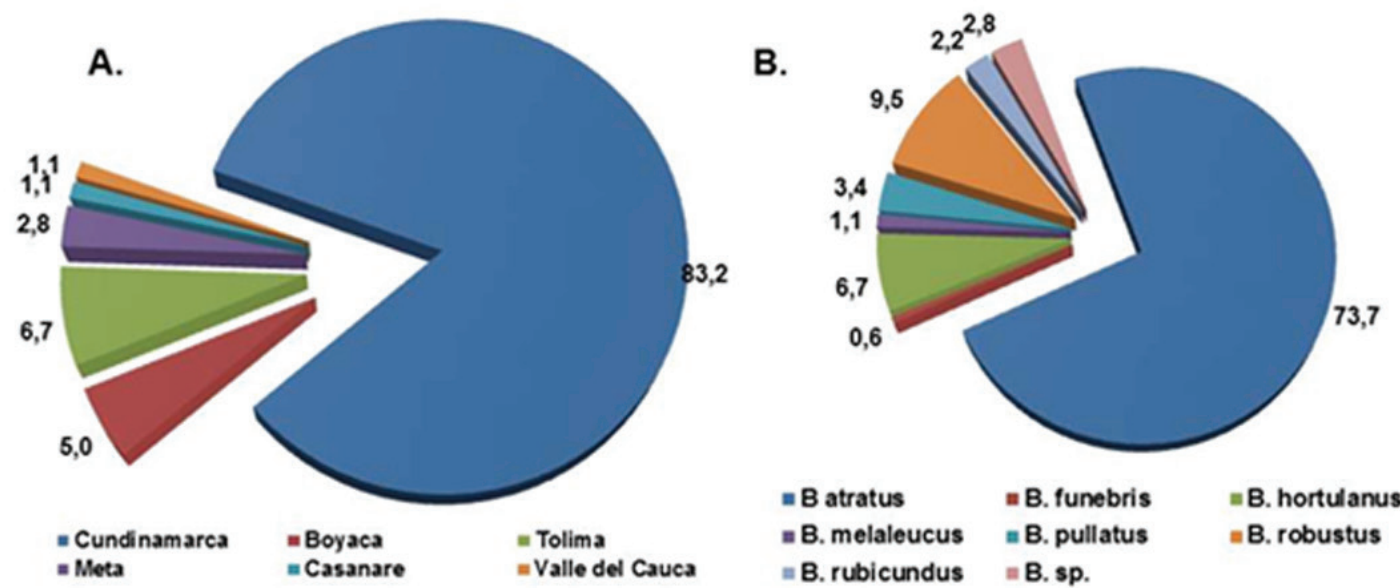

C.
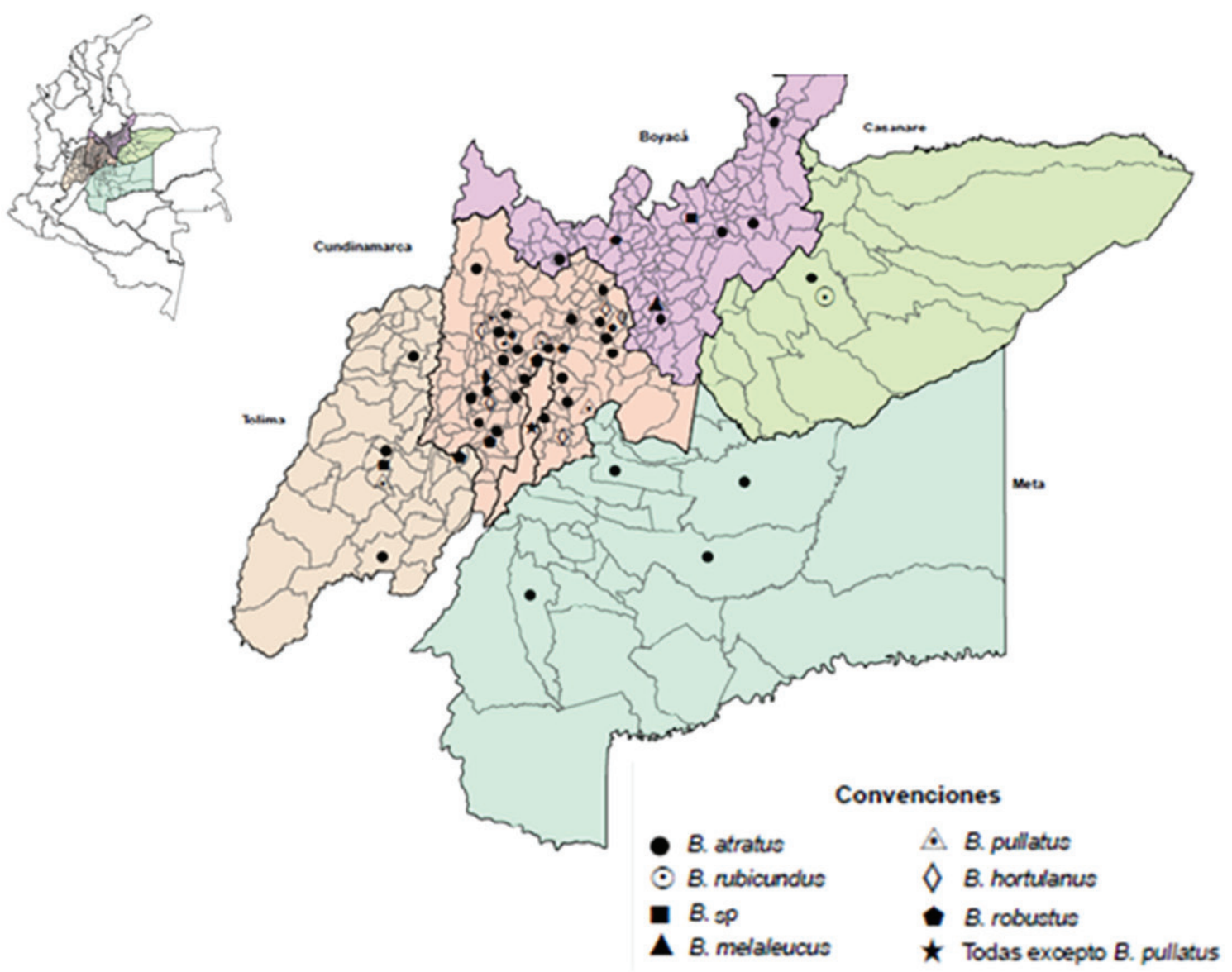

Figura 1. Distribución de las especies de Bombus de la colección entomológica de la U.D.C.A. A. Porcentaje según la cantidad de especímenes registrados por departamento; B. Porcentaje de la cantidad de especímenes según la especie; C. Distribución de las especies encontradas en la colección dentro de cinco departamentos ubicados en la zona central de Colombia. 
lectó en tres departamentos y en diez localidades (18,2\%). De $B$. hortulanus, se colectaron 15 especímenes $(6,7 \%)$. $B$. hortulanus solamente se colectó en un departamento y en nueve localidades $(16,4 \%)$. De $B$. pullatus, se encontraron siete especímenes $(3,4 \%)$, en dos departamentos y en tres localidades $(5,5 \%)$, frecuencia que no concuerda con lo descrito por Nates-Parra (2005), donde se menciona que es una especie abundante. De $B$. rubicundus, se colectaron cinco especímenes (2,2\%), en dos departamentos y cuatro localidades (7,3\%). De B. melaleucus, se colectaron dos especímenes $(1,1 \%)$, en dos departamentos y dos localidades (3,6\%), lo que contribuye a calificarla como especie rara, ya que se encuentra muy poco en las colecciones entomológicas (Nates-Parra, 2005). De la morfo-especie, designada como Bombus sp., se colectaron siete especímenes (2,8\%), en tres departamentos y cuatro localidades (7,3\%) (Figura 1B). Estas cifras, de la mayor o menor abundancia, procedentes de diferentes localidades son importantes para iniciar trabajos dirigidos a establecer los mapas de distribución de las especies, utilizando sistemas de información geográfica (SIG), con el propósito de conocer en detalle su ubicación y los factores que puedan estar regulando las poblaciones.
En la tabla 1, se muestra la distribución altitudinal de las especies de Bombus presentes en la colección entomológica de la U.D.C.A. En los registros, se encontró información para 177 (83,1\%) especímenes. Las especies B. atratus, B. hortulanus, $B$. robustus, $B$. rubicundus y $B$. sp., se evidenciaron en todos los rangos de altitud, que comprendieron 0-1000, 1001 a 2000 y 2001 a 3000msnm. De B. funebris, solamente se registró un espécimen, colectado en el rango de altitud de 2001 a 3000msnm, lo que concuerda con Nates-Parra (2005), en donde se menciona que esta especie es la que más resiste las grandes altitudes. De $B$. pullatus, se colectaron seis especímenes, en los rangos de altitud de 0-1000 y de 1001 a 2000msnm y de $B$. melaleucus, se capturaron dos especímenes, en los rangos de altitud de 1001 - 2000 y de 2001 a 3000msnm. Estos resultados de la distribución altitudinal requieren de mayor número de observaciones y son importantes, porque indican el valor que las especies de Bombus pueden tener en la polinización de cultivos y la flora silvestre en los diferentes pisos térmicos, presentes en la geografía de Colombia, que abarca los Andes, los valles interandinos, la costa y los llanos.

Tabla 1. Distribución altitudinal del número de especímenes de las especies de Bombus presentes en la colección entomológica de la U.D.C.A.

\begin{tabular}{|c|c|c|c|c|c|c|c|c|}
\hline $\begin{array}{c}\text { Altitud } \\
(\mathrm{msnm})\end{array}$ & $\begin{array}{c}\text { B. } \\
\text { atratus }\end{array}$ & $\begin{array}{c}\text { B. } \\
\text { funebris }\end{array}$ & $\begin{array}{c}\text { B. } \\
\text { hortulanus }\end{array}$ & $\begin{array}{c}\text { B. } \\
\text { pullatus }\end{array}$ & $\begin{array}{c}\text { B. } \\
\text { melaleucus }\end{array}$ & $\begin{array}{c}\text { B. } \\
\text { robustus }\end{array}$ & $\begin{array}{c}\text { B. } \\
\text { rubicundus }\end{array}$ & B. sp \\
\hline $0-1000$ & 15 & & 2 & 4 & & 1 & 1 & 2 \\
\hline $1001-2000$ & 24 & & 2 & 2 & 1 & 5 & 1 & 1 \\
\hline $2001-3000$ & 90 & 1 & 8 & & 1 & 11 & 2 & 2 \\
\hline Mínimo & 178 & 2630 & 799 & 506 & 1757 & 323 & 350 & 506 \\
\hline Máximo & 3000 & 2630 & 2713 & 1895 & 2630 & 2630 & 2630 & 2360 \\
\hline
\end{tabular}

No se encontraron registros de especímenes colectados a altitudes mayores de 3000msnm, lo que significa que se requieren realizar muestreos en zonas de estas altitudes, para establecer las especies que las habitan y los hábitos y uso de flora de las mismas. Los estudios futuros deben establecer la abundancia y la presencia de estas abejas, ya que han sido denominadas las abejas de los páramos (Nates-Parra \& Gonzáles, 2000) y juegan un papel importante en la conservación de estos ecosistemas. Igualmente, se debe confirmar si los abejorros del género Bombus se presentan a altitudes bajas, como lo registra la información de las colectas, puesto que, corrientemente, se indica que las poblaciones de estos insectos, en el trópico, se muestran, principalmentem en ecosistemas de alta montaña (Michener \& Amir, 1977; Nates-Para et al. 2006).
En cuanto a la información sobre la forma de colecta, de los 213 especímenes, $145(68,1 \%)$ presentaron información y 68 no tenían datos (30,9\%). El material con información permitió establecer que 46 especímenes (21,6\%), se colectaron polinizando flores y 99 especímenes (46,5\%) presentaron información incompleta, como: visitando flores $(n=11 ; 5,2 \%)$, de los cuales, no se indicó la identificación de la planta; follaje $(n=18 ; 8,5 \%)$, tampoco se indicó la identificación de la planta; encontrados en el suelo $(n=4 ; 1,9 \%)$ y colectados al vuelo ( $n=66 ; 31,0 \%)$. Estos dos últimos aspectos claramente indican la necesidad de prestar atención a los hábitos y a las relaciones que establece Bombus con las plantas que visitan y florecen a nivel del suelo, como los tréboles o diente de león, entre otras, donde además los individuos de Bombus se pueden encontrar en las flores, descansando o volando 
a ras del suelo, buscando alimento. La falta de información demuestra, claramente, el descuido al realizar la colección, lo que indica que es esencial instruir, de manera adecuada, a los estudiantes cuando realizan colecciones, para garantizar que sean rigurosos en el registro de la información, ya que es de valor para el uso de la colección, como herramienta de educación e investigación. Los polinizadores, por sus hábitos de alimentación, cuando visitan una especie de planta que está en floración, corrientemente repiten varias visitas, por lo que ofrecen la oportunidad al estudiante de observar el fenómeno de la polinización, colectar la información y unos especímenes para cumplir con el requisito del curso y, a su vez, aportar datos para la investigación y conservación de este grupo.

Sobre las plantas hospedantes, se encontraron 46 registros de Bombus visitando 28 especies de plantas. Estos registros corresponden a solamente tres especies de Bombus, de las ocho registradas en la colección. Las otras cinco especies de Bombus, se capturaron al vuelo o no presentaron información de captura. $B$. atratus fue la especie con mayor frecuencia de registros de plantas visitadas $(n=39 ; 84,8 \%)$; $B$. hortulanus, se encontró en cuatro $(8,7 \%)$ y B. robustus, únicamente se registró visitando tres $(6,5 \%)$ plantas (Tabla 2). Ninguna planta se encontró que fuera visitada por todas las especies de Bombus.

La mora (Rubus sp., Rosaceae) fue la planta que registró el mayor número de especies de Bombus, como polinizadores. Se registró que es utilizada por tres especies (Tabla 2). La mora es una planta silvestre, que demuestra que es muy importante para las especies de Bombus; tiene amplia distribución en Colombia y crece en bordes de vías, corrientes de agua y el sotobosque, lo que la hace una candidata para propagación en los programas de conservación de Bombus.

$B$. atratus fue la única especie que registró frecuencia de colecta de cinco, tres, dos y una, mientras que las otras dos especies de Bombus solamente presentaron frecuencia de una colecta sobre las plantas visitadas (Tabla 2). Por especie de planta, el mayor número de frecuencia de colectas, cinco, se realizó en frijol y en trébol, seguido por tres colectas, sobre guayaba, fresa y papa. La papa y el trébol presentaron frecuencias de dos y las demás plantas, de uno. Considerar la frecuencia de las colectas es importante, porque demuestra la preferencia de las especies de Bombus, por determinados recursos florales (Tabla 2).

La colecta de las especies de Bombus en las plantas también indica que se debe evaluar no solamente el beneficio para el polinizador sino también el servicio de la polinización y el riesgo que enfrenta Bombus cuando estas plantas son cultivos, como la papa y la fresa, en los que se aplican, con frecuencia insecticidas, para el manejo de plagas. Se encontró que $B$. atratus muestra preferencia por visitar estos cultivos (Tabla 2), lo que puede afectar sus poblaciones. En papa, la polinización no es indispensable para la producción de semilla, ya que la que se utiliza comercialmente son los tubérculos, mientras que en la fresa, la polinización cruzada es indispensable para obtener alta producción y frutos de buena calidad. La consideración de la presencia de Bombus en estos cultivos demuestra la necesidad de desarrollar programas de manejo de los polinizadores, que aseguren el servicio de polinización y la sobrevivencia en el medio.

Por familia de plantas, el mayor número de registros de especies lo presentó, con cinco, la familia Rosaceae (ciruelo, durazno, fresa, mora y rosa) y la familia Asteraceae (dalia, diente de león, girasol, margarita y botón de oro), seguidas por la Fabaceae, con cuatro especies (arveja, frijol, trébol y trébol rojo). Finalmente, las solanáceas, con tres especies (ají, papa y tomate), en las cuales, la actividad del zumbido de Bombus en las flores se puede percibir con claridad, para hacer desprender el polen de las anteras, que son fusionadas (Delaplane \& Mayer, 2000; Almanza, 2007; Aldana et al. 2007).

Los resultados que se presentan sobre la flora indican que existe necesidad de recalcar la importancia de registrar las plantas hospedantes. Los registros de las plantas hospedantes solamente alcanzaron el $21,6 \%$ de la información, que debe contener el material colectado y únicamente dos registros presentaron los nombres científicos de las plantas (Tabla 2). La información sobre la flora es esencial para adelantar programas de manejo y conservación de las poblaciones de Bombus. El colector debe establecer que el insecto que se colecta está visitando las flores, ya sea para obtener polen o néctar, lo que le permite conocer y comprobar que es un polinizador. Al material colectado, se le deben colocar los nombres científicos y vulgares de las plantas, para tener una mayor exactitud sobre esta información.

El estado de curaduría de los especímenes, se inició con un valor promedio de tres, un mínimo de uno, para especímenes que no presentaron ninguna información y máximo de cuatro, para especímenes que presentaban información, pero que requerían curaduría. El mayor limitante consistió en que los especímenes mostraban información faltante de localidad, fecha de colección, planta hospedante y colector. Además, se hallaron dispersos en diferentes gabinetes, cajas y en viales con alcohol, sin ningún dato, que permitiera establecer dónde encontrarlos e, igualmente, no existían registros ni en tarjetas o en bases de datos, para el manejo de la información. En general, lo especímenes estaban mezclados con otros insectos, exhibían pérdida de partes y presencia de plagas y hongos, por lo que el valor de la curaduría fue bajo.

La colección expone especímenes colectados desde 1994 
Tabla 2. Registro de la frecuencia de las plantas hospedantes de las especies de Bombus depositadas en la colección de insectos de la U.D.C.A.

\begin{tabular}{|c|c|c|c|c|c|c|c|c|c|c|c|}
\hline Plantas & $\begin{array}{l}\text { Nombre } \\
\text { científico }\end{array}$ & Familia & $\begin{array}{l}\text { Bombus } \\
\text { atratus }\end{array}$ & $\begin{array}{l}\text { Bombus } \\
\text { funebris }\end{array}$ & $\begin{array}{c}\text { Bombus } \\
\text { hortu- } \\
\text { lanus }\end{array}$ & $\begin{array}{l}\text { Bombus } \\
\text { melaleu- } \\
\text { cus }\end{array}$ & $\begin{array}{l}\text { Bombus } \\
\text { pullatus }\end{array}$ & $\begin{array}{l}\text { Bombus } \\
\text { robustus }\end{array}$ & $\begin{array}{l}\text { Bombus } \\
\text { rubicun- } \\
\text { dus }\end{array}$ & $\begin{array}{c}\text { Bombus } \\
\text { sp. }\end{array}$ & Total \\
\hline Dalia & Dahlia spp. & Asteraceae & 0 & 0 & 0 & 0 & 0 & 1 & 0 & 0 & 1 \\
\hline Diente león & $\begin{array}{l}\text { Taraxacum } \\
\text { officinale }\end{array}$ & Asteraceae & 1 & 0 & 0 & 0 & 0 & 0 & 0 & 0 & 1 \\
\hline Girasol & $\begin{array}{l}\text { Helianthus } \\
\text { annuus }\end{array}$ & Asteraceae & 1 & 0 & 0 & 0 & 0 & 0 & 0 & 0 & 1 \\
\hline Margarita & $\begin{array}{l}\text { Chrysanthe- } \\
\text { mum sp }\end{array}$ & Asteraceae & 1 & 0 & 0 & 0 & 0 & 0 & 0 & 0 & 1 \\
\hline Botón de oro & $\begin{array}{l}\text { Thytonia di- } \\
\text { versifolia }\end{array}$ & Asteraceae & 1 & 0 & 0 & 0 & 0 & 0 & 0 & 0 & 1 \\
\hline Besito & $\begin{array}{l}\text { Impatiens } \\
\text { balsamina }\end{array}$ & \begin{tabular}{|l} 
Balsami- \\
naceae
\end{tabular} & 1 & 0 & 0 & 0 & 0 & 0 & 0 & 0 & 1 \\
\hline Achira & Canna spp. & Cannaceae & 0 & 0 & 0 & 0 & 0 & 1 & 0 & 0 & 1 \\
\hline Batatilla & Ipomoea spp. & \begin{tabular}{|l|} 
Convolvu- \\
laceae
\end{tabular} & 1 & 0 & 0 & 0 & 0 & 0 & 0 & 0 & 1 \\
\hline Calabacín & Cucurbita sp. & Cucurbitaceae & 1 & 0 & 0 & 0 & 0 & 0 & 0 & 0 & 1 \\
\hline Arveja & \begin{tabular}{|l|} 
Pisum sati- \\
vum
\end{tabular} & Fabaceae & 1 & 0 & 0 & 0 & 0 & 0 & 0 & 0 & 1 \\
\hline Fríjol & $\begin{array}{l}\text { Phaseolus } \\
\text { vulgaris }\end{array}$ & Fabaceae & 5 & 0 & 0 & 0 & 0 & 0 & 0 & 0 & 5 \\
\hline Trébol & Trifoium sp. & Fabaceae & 5 & 0 & 1 & 0 & 0 & 0 & 0 & 0 & 6 \\
\hline Trébol rojo & $\begin{array}{l}\text { Trifolium } \\
\text { pratenses }\end{array}$ & Fabaceae & 2 & 0 & 0 & 0 & 0 & 0 & 0 & 0 & 2 \\
\hline Tulipan & Tulipa spp & Liliaceae & 1 & 0 & 0 & 0 & 0 & 0 & 0 & 0 & 1 \\
\hline Cayeno & Hibiscus & Malvaceae & 0 & 0 & 1 & 0 & 0 & 0 & 0 & 0 & 1 \\
\hline Guayaba & Psium guaba & Myrtaceae & 3 & 0 & 0 & 0 & 0 & 0 & 0 & 0 & 3 \\
\hline Ciruelo & Prunus spp. & Rosaceae & 1 & 0 & 0 & 0 & 0 & 0 & 0 & 0 & 1 \\
\hline Durazno & $\begin{array}{l}\text { Prunus per- } \\
\text { sica }\end{array}$ & Rosaceae & 1 & 0 & 0 & 0 & 0 & 0 & 0 & 0 & 1 \\
\hline Fresa & $\begin{array}{l}\text { Fragaria } \\
\text { vesca }\end{array}$ & Rosaceae & 3 & 0 & 1 & 0 & 0 & 0 & 0 & 0 & 4 \\
\hline Mora & Rubus spp. & Rosaceae & 2 & 0 & 1 & 0 & 0 & 1 & 0 & 0 & 4 \\
\hline Rosa & Rosa spp. & Rosaceae & 1 & 0 & 0 & 0 & 0 & 0 & 0 & 0 & 1 \\
\hline Naranja & Citrus sp & Rutaceae & 2 & 0 & 0 & 0 & 0 & 0 & 0 & 0 & 2 \\
\hline Ají & $\begin{array}{l}\text { Solanum } \\
\text { capsicum }\end{array}$ & Solanaceae & 1 & 0 & 0 & 0 & 0 & 0 & 0 & 0 & 1 \\
\hline Papa & \begin{tabular}{|l}
$\begin{array}{l}\text { Solanum tu- } \\
\text { berosus }\end{array}$ \\
\end{tabular} & Solanaceae & 3 & 0 & 0 & 0 & 0 & 0 & 0 & 0 & 3 \\
\hline Tomate & \begin{tabular}{|l|} 
Lycopersi- \\
cum esculen- \\
tum
\end{tabular} & Solanaceae & 1 & 0 & 0 & 0 & 0 & 0 & 0 & 0 & 1 \\
\hline Subtotal & & & 39 & 0 & 4 & 0 & 0 & 3 & 0 & 0 & 46 \\
\hline Porcentaje & & & 84,8 & 0 & 8,7 & 0 & 0 & 6,5 & 0 & 0 & \\
\hline Flor & & & 8 & 0 & 1 & 0 & 0 & 2 & 0 & 0 & 11 \\
\hline Follaje & & & 16 & 0 & 0 & 0 & 0 & 0 & 2 & 0 & 18 \\
\hline Suelo & & & 1 & 1 & 0 & 0 & 1 & 0 & 1 & 0 & 4 \\
\hline Vuelo & & & 42 & 1 & 2 & 2 & 5 & 7 & 2 & 5 & 66 \\
\hline $\begin{array}{l}\text { Información } \\
\text { incompleta }\end{array}$ & & & 67 & 2 & 3 & 2 & 6 & 9 & 5 & 5 & 99 \\
\hline Porcentaje & & & 67,7 & 2,0 & 3,0 & 2,0 & 6,1 & 9. & 5,1 & 5,1 & \\
\hline Sin información & & & 49 & 0 & 8 & 0 & 1 & 8 & 0 & 2 & 68 \\
\hline Porcentaje & & & 72,1 & 0 & 11,8 & 0 & 1,5 & 11,8 & 0 & 2,9 & \\
\hline Total & & & 155 & 2 & 15 & 2 & 7 & 20 & 5 & 7 & 213 \\
\hline Porcentaje & & & 72,8 & 0,9 & 7,0 & 0,9 & 3,3 & 9,4 & 2,3 & 3,3 & 100 \\
\hline
\end{tabular}


hasta el 2012. Los únicos años donde no se recolectaron Bombus fueron 1995 y 1996. B. atratus, se colectó continuamente desde 1997 hasta el 2011. La colección de las otras especies fue muy variable a través del tiempo y en un bajo número. El año de mayor número de colectas fue el 2012, posiblemente, por el interés despertado con la realización del presente trabajo, que acompañó a los estudiantes al campo.

La colección de Bombus de la U.D.C.A, se organizó y los especímenes se colocaron en gabinetes identificados por orden (Hymenoptera), familia (Apidae) y tribu (Bombini). Dentro del gabinete, el material se dispuso por especie y la información de cada espécimen, se registró en la etiqueta. Las genitalias y las cámaras del agujón extraídas para el proceso de identificación, se dejaron adheridas al espécimen, ubicadas dentro de viales, para su conservación y posterior uso, en otros trabajos de identificación. La colección de Bombus de la U.D.C.A, se dejó preparada por especies en orden filogenético, de acuerdo con Michener (2007).

El valor de curaduría de la colección de Bombus de la Universidad, se restauró a un nivel de 9, ya que se rescató la información geográfica, ecológica, colectores y fechas. Adicionalmente, esta actualización permitió realizar investigaciones con el material, tomar datos, fotos, plasmar dibujos, mediciones, descripciones para publicaciones y estudios ecológicos, como la identificación de las especies de Bombus de la colección entomológica de la U.D.C.A (Téllez \& Posada, en preparación).

Se recomienda que para realizar la colección de material entomológico, se deben exigir a los participantes la información que deben contener los especímenes. Adicionalmente, recalcar la necesidad que los especímenes de Bombus sean bien montados en alfileres, con el propósito que el material sea de valor para la colección, de lo contrario, no se justifica colectar. Los individuos de Bombus y otras especies que se caracterizan por presentar pilosidad en todo su cuerpo, no se deben almacenar en alcohol, ya que cuando se secan, la pilosidad queda pegada al cuerpo, lo que puede llegar a dificultar la identificación, además de que, estéticamente, no queda presentable el insecto.

Revisar las colecciones del país, con el propósito conocer en detalle el registro de las especies de Bombus y conocer los aspectos de distribución y plantas hospedantes, aportaría información valiosa para el uso de las diferentes especies, en la polinización de cultivos y su conservación. Estas visitas, se deben complementar con colectas en sitos geográficos diferentes a la región central del país (Cundinamarca), para tener un mayor conocimiento de esta fauna.

Promover el conocimiento de las plantas hospedantes de Bombus, con el objetivo de cultivarlas o preservarlas en el medio para mantener las poblaciones de estos polinizadores, puede ser una iniciativa pionera para la conservación de este género de abejas.

Agradecimientos: A la U.D.C.A, por el contrato de joven investigador concedido a Lorena Téllez Farfán, para adelantar este trabajo de curaduría de los especímenes de Bombus de la colección entomológica de la Facultad de Ingeniería Agronómica. Conflicto de intereses: El manuscrito fue preparado y revisado con la participación de todos los autores, quienes declaramos que no existe ningún conflicto de intereses que ponga en riesgo la validez de los resultados presentados.

\section{BIBLIOGRAFÍA}

1. ABRAHAMOVICH, A.; DÍAZ, N. 2002. Bumble bees of the Neotropical Region (Hymenoptera: Apidae). Biota Col. 3(2):199-214.

2. ALDANA, J.; CURE, J.R.; ALMANZA, M.T.; VECIL, D.; RODRÍGUEZ, D. 2007. Efecto de Bombus atratus (Hymenoptera: Apidae) sobre la productividad de tomate (Lycopersicon esculentum Mill.) bajo invernadero en la Sabana de Bogotá, Colombia. Agr. Col. 25(1):62-71.

3. ALLSOPP, M.H.; DE LANGE, W.J.; VELDTMAN, R. 2008. Valuing Insect Pollination Services with Cost of Replacement. PLoS ONE 3(9): e3128. Disponible desde internet en http://www.plosone.org/article/ info:doi/10.1371/journal.pone.0003128 (con acceso 23/01/2013).

4. ALMANZA, M.T. 2007. Management of Bombus atratus bumblebees to pollinate Lulo (Solanum quitoense L.), a native fruit from the Andes of Colombia. Ecology and development Series No. 50. 127p.

5. BROWN, M.J.F.; PAXTON, R.J. 2009. The conservation of bees: a global perspective. Apidologie.40:410-416.

6. CHUNG, K.K. 1993. Biodiversity, conservation and inventory: why insects matter. Biodiv. \& Conserv. 3(2): 191-214.

7. CRUZ, B.C.A.; POSADA F., F. 2003. Evaluación de la biodiversidad de la zona cafetera colombiana representada en la colección de artrópodos de Cenicafé. Rev. Col. Entomol. 29(2):107-112.

8. CRUZ, P.; ESCOBAR, A.; ALMANZA, M.T.; CURE, J.R. 2008. Implementación de mejoras para la cría en cautiverio de colonias del abejorro nativo Bombus 
pauloensis (= B. atratus) (Hymenoptera: Apoidea). Rev. Fac. Cienc. Bás. U. Militar Nueva Granada. 4(1):70-83.

9. DELAPLANE, K.; MAYER, D. 2000. Crop pollination by bees. CABI. New York, USA. 334p.

10. HERNÁNDEZ, M.P.; BELLOTTI, A.C.; CARDONA, C.; LAPOINTE, S.; PANTOJA, A. 1995. Organización y Utilidad de una Colección de Insectos para referencia y en Cuatro Cultivos Tropicales. Rev. Col. Entomol. 21(1):59-62.

11. JAMES, R. 2010. Biology, News from the USDA-ARS Pollination Insects-Biology, Management and Systematics Research Unit in Logan, UT Utah Pest News. 4: 8-9. Disponible desde internet en http://utahpests. usu.edu/files/uploads/UtahPests-Newsletter-summer10.pdf (con acceso 23/01/2013).

12. KLEIN, A.M.; VAISSIÈRE, B.E.; CANE, J.H.; STEFFANDEWENTER, I.; CUNNINGHAM, S.A.; KREMEN, C.; TSCHARNTKE, T. 2007. Importance of pollinators in changing landscapes for world crops. Proc. R. Soc. Lond. B, Biol. Sci., 274:303-313. Disponible desde internet en: http://rspb.royalsocietypublishing. org/citmgr?gca= royprsb;274/1608/303 (con acceso 23/01/2013).

13. LIÉVANO, A.; OSPINA, R.; NATES-PARRA, G. 1994. Contribución al conocimiento de la taxonomía del género Bombus en Colombia (Hymenoptera: Apidae). Trianea. 5:221-223.

14. McGINLEY, R. 1993. Where's the management in collections management? Library of Congress. Vol. 3. Washington, USA. p.309-338.

15. MICHENER, C.D. 2007. Bees of the World. 2nd Ed. The Johns Hopkins University Press. USA. 953p.

16. MICHENER, C.D.; AMIR, M. 1977. The seasonal cycle and habitat of a tropical bumble bee. Pacific Insects. 17(2-3):237-240.

17. MORALES-ROZO, C.; ROJAS-PRIETO, D.; CURE-HAKIM, J. 2006. Actividad forrajera de un nido silvestre de Bombus atratus Franklin (Hymenoptera: Apidae) en la Sabana de Bogotá. p. 49. En: III Encuentro Colombiano de Abejas Silvestres. 2006. Laboratorio de Investigaciones en Abejas LABUN, Universidad Nacional de Colombia. Santa Marta, Colombia.

18. NATES-PARRA, G. 2005. Abejas corbiculadas de Colombia, Hymenoptera: Apidae. Universidad Nacional de Colombia. Bogotá, Colombia. 156p.

19. NATES-PARRA, G.; GONZÁLES, V. 2000. Las abejas silvestres de Colombia: por qué y cómo conservarlas. Acta Biol. Col. 5(2): 1-33.

20. NATES-PARRA, G.; PARRA, A.; RODRÍGUEZ, A.; VAQUERO, P.; VÉLEZ, D. 2006. Abejas silvestres (Hymenoptera: Apoidea) en ecosistemas urbanos: Estudio en la ciudad de Bogotá y sus alrededores. Rev. Col. Entomol. 32(1):77-84.

21. OTTERSTATTER, M.C.; THOMSON, J.D. 2008. Does Pathogen Spillover from Commercially Reared Bumble Bees Threaten Wild Pollinators? PLoS ONE 3(7): e2771. Disponible desde internet en http://www.plosone.org/article/info\%3Adoi\%2F10.1371\%2Fjournal. pone.0002771 (con acceso 11/08/2012).

22. PARK, M.G.; ORR, M.C.; DANFORTH, B.N. 2010. The Role of Native Bees in Apple Pollination. New York Fruit Quarterly 18(1):21-25. Disponible desde internet en http://www.nyshs.org/pdf/fq/10spring/therole-of-native-bees-in-apple-pollination.pdf (con acceso 23/01/2013).

23. PRŶS-JONES, O.; CORBET, S. 1991. Bumblebees. The Richmond Publishing Co. Ltd. England. 92p.

24. TÉLLEZ-FARFÁN, L.; POSADA-FLÓREZ, F.J. Identificación de especies de Bombus de la colección de insectos de la U.D.C.A. En preparación.

25. TRIPPLEHORN, C.A.; JOHNSON, N.F. 2005. Borror and DeLong's Introduction to the Study of Insects, 7th edition. Thomas Brooks/Cole. USA. 864p.

Recibido: Julio 12 de 2012

Aceptado: Junio 5 de 2013

Como citar:

Téllez-Farfán, L.; Posada-Flórez, F.J. 2013. Curaduría e identificación de la fauna de bombus de la colección de entomología de la Universidad de Ciencias Aplicadas y Ambientales U.D.C.A. Rev. U.D.CA Act. \& Div. Cient. 16(2): 501-509. 